The following paper was presented at The 9th Workshop on Disfluency in Spontaneous Speech (DiSS 2019) held at ELTE Eötvös Loránd University in Budapest, Hungary on 12-13 September, 2019.

Title: $\quad$ Halt command in word retrieval

Author(s): $\quad$ Mária Gósy

Abstract: $\quad$ In this study, occurrences and temporal patterns of five types of disfluencies were analyzed that show a common feature on the surface. All of them have some kind of interruption of content words followed by some continuation. The purpose was to show whether the place of interruption of the word articulation and the durational patterns of the editing phases are characteristic of re-starts, false starts, slips of the tongue, pauses within words, and prolongations. More than 1,400 instances were processed. Both (i) the number of pronounced segments of abandoned words and the duration of the corresponding editing phases are characteristic of a specific disfluency type, and (ii) speakers select a strategy to overcome their speech planning difficulties most economically.

DOI: https://doi.org/10.21862/diss-09-002-gosy

Citation (JIPA): Gósy, Mária. 2019. Halt command in word retrieval. In: R. L. Rose \& R. Eklund (eds.), Proceedings of DiSS 2019, The 9th Workshop on Disfluency in Spontaneous Speech, 12-13 September, 2019, Budapest, Hungary, 3-6.

The complete proceedings for DiSS 2019 are available as follows.

ISBN: 978-963-489-063-8

DOI: $\quad$ https://doi.org/10.21862/diss-09

DiSS 2019 was sponsored by The Faculty of Humanities, ELTE Eötvös Loránd University and the International Speech Communication Association (ISCA).
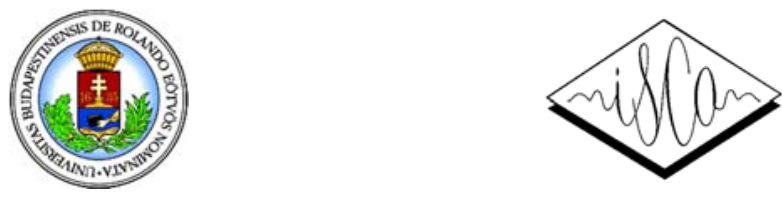


\title{
Halt command in word retrieval
}

\author{
Mária Gósy \\ Research Institute for Linguistics, Hungarian Academy of Sciences, and \\ Department of Phonetics, ELTE University, Budapest, Hungary
}

\begin{abstract}
In this study, occurrences and temporal patterns of five types of disfluencies were analyzed that show a common feature on the surface. All of them have some kind of interruption of content words followed by some continuation. The purpose was to show whether the place of interruption of the word articulation and the durational patterns of the editing phases are characteristic of re-starts, false starts, slips of the tongue, pauses within words, and prolongations. More than 1,400 instances were processed. Both (i) the number of pronounced segments of abandoned words and the duration of the corresponding editing phases are characteristic of a specific disfluency type, and (ii) speakers select a strategy to overcome their speech planning difficulties most economically.
\end{abstract}

\section{Introduction}

A wide variety of errors-like conceptual errors, syntactic errors, lexical errors, phonemic/phonetic (Ph-) errors, prosodic errors, morphemic errors, errors relating to social context (etc.) - and other types of disfluencies-like filled pauses, prolongations, repetitions (etc.) - can occur when people speak spontaneously (Levelt, 1989; Postma, 2000). Although the types and occurrences of disfluencies are different across speakers, speech styles, and languages, practically all speakers produce them. The speech monitor is a welldeveloped mechanism that is sensitive to several processes and their outcomes during speech planning and execution, and makes it possible for the speaker to prevent, detect and repair real errors. Although there is no accomplished consensus concerning the nature and character of the process of internal monitoring (e.g. Levelt, 1989; Huettig \& Hartsuiker, 2010), everybody accepts the existence of such a monitor having a definite task during speech production. Real erroneous words and those that are judged by the speaker to be erroneous are sometimes interrupted on the surface as a result of a 'halt command' the articulatory organs receive. The halt command is preceded by some discrepancy of intended and monitored speech calling for some context-dependent solution.

Self-initiated self-interruptions are defined as the halting of the continuous articulation of a word at a certain point. These interruptions follow the classical process: A speaker interrupts the flow of speech at an 'interruption point' to repair (or modify) something $\mathrm{s} /$ he has said before, and/or monitors the produced speech prior to halting (Levelt, 1989; Nooteboom \& Quené, 2008). The stretch of speech to be repaired is the 'reparandum' while the correction is the 'repair'. The 'repair' sometimes does not contain any change or modification, like in the case of re-starts. The editing phase is frequently a silent period or a filled pause, or may contain cue phrases, or any combination of these. The editing phase can be completely missing when the repair immediately follows the reparandum. In this study we wanted to find answers for the interrelations between certain disfluencies and interruption points (i.e. the number of pronounced segments before halting) as well as between these disfluencies and the durations of their editing phases. Hungarian is an agglutinating language, so content words can easily consist of 10 or more segments (frequently more than 5 syllables) due to suffixation, a fact that provides an excellent possibility to analyze the various numbers of segments before halting.

In the line of Blackmer and Mitton's (1991) view we claim that many disfluencies are edited errors. Edited errors concern $\mathrm{Ph}$-errors and false starts where the errors appear on the surface and should be repaired. Although the notion of editing phase is rather well defined in the literature, we will interpret it in a broad sense as any period between the interruption of word articulation and its continuation, whether it is implemented as silent pause, filled pause, some cue phrase, nothing (immediate continuation), or even some unexpected prolongation of a segment.

There are disfluency phenomena where both reparandum and repair are assumed to exist, but no surface error can be detected. Editing phases in these cases are supposed to refer to monitoring and finding a strategy for problem solution during speech planning. These disfluency phenomena are (among others) re-starts, pauses within words (PwW) and prolongations. In the case of re-starts there is no erroneous part of the word, but the speaker senses a possible error during monitoring. The monitoring and the supposed preparedness for repair require extra time resulting in an editing phase between halting and continuation. Monitoring terminates with 
the conclusion that no error could be found. So, the pronounced part of the word will be repeated, and word retrieval and its articulation completed. A very similar process takes place in the case of the phenomenon of PwW. The speaker interrupts word articulation and after a while there is a continuation resulting in completing the word. The difference between re-starts and PwWs is the lack of repetition of the reparandum in the PwW cases. We suggest that there should be a third variant of this process with an editing phase that manifests itself in segment prolongation. Interruptions of the speech flow within a word may refer to various problems that exist in any phase of speech planning, lexical access and pronunciation. Figure 1 illustrates our broadened conception of the editing phase.

\begin{tabular}{|c|c|c|}
\hline reparandum & $\begin{array}{c}\text { editing } \\
\text { phase }\end{array}$ & repair \\
\hline $\begin{array}{l}\text { mo 'ci' } \\
\text { szo 'roo' } \\
\text { lövény 'blant' } \\
\text { város 'city' } \\
\text { abl }\end{array}$ & $\begin{array}{l}\text { pause } \\
\text { pause } \\
\text { pause } \\
\text { pause } \\
\text { aaa }\end{array}$ & $\begin{array}{l}\text { mozi 'cinema' } \\
\text { konyha 'kitchen' } \\
\text { növény 'plant' } \\
\text { ba 'to' as suffix } \\
\text { kok 'windooows' }\end{array}$ \\
\hline
\end{tabular}

Figure 1. The structure of error, interruption point, editing phase and repair for re-starts, false starts, slips, PwWs, prolongations.

We examined five types of disfluencies from the aspects of repair processes, those involving real errors (false starts and Ph-errors) and those involving no surface errors but showing interruption and completion of the words (re-starts, PwWs and prolongations) indicating some kind of covert error during speech planning. Trouble signals are not confirmed in the latter cases and the speakers select from the possible simplest solutions in the given context to finish word articulation. The questions arise: (i) Is the place of interruption characteristic of the disfluency in question? (ii) Do durations of the editing phases reflect monitoring and repair processes in these five disfluencies irrespective of the source of the trouble behind them? Our purpose was to show whether the place of interruption and durations of the editing phases would shed light on the specific properties of the analyzed disfluencies.

We had three hypotheses. (1) Halt command at a definite time point in word articulation would reflect the type of disfluency. (2) Editing phase duration is characteristic of disfluency type. (3) There will be a close interrelation between the quantity of articulated segments of abandoned words and the durations of the editing phases.

\section{Methodology}

Spontaneous narratives and conversations of 52 Hungarian-speaking subjects (aged between 20 and 40 years, half of them were females) were randomly selected (with the exceptions of age and gender) from the BEA Spontaneous Speech Database of Hungarian (Gósy, 2012). All speakers had normal hearing, and none of them had any speech defects. All of them had a similar socio-economic status.

Speakers were asked to speak about their family, life, hobby, to share their opinion on a specific topic raised by the interviewer, and to summarize two short stories they heard during the interview. In addition, a 3-member conversation about various topics with each participant was subjected to analysis. A total of 27.7 hours of spontaneous speech material was analyzed. The average length of the speech material per speaker was about 30 minutes. Five types of disfluencies were identified with all speakers (their occurrences showed large individual differences). Altogether 1,472 disfluencies were identified in the corpus, and about 28 instances could be found per speaker (also with large differences across subjects). Disfluencies that concerned content words were considered.

All editing phases were manually annotated in the waveform and spectrogram displays via continuous listening to the words in Praat (Boersma \& Weenink, 2014). Durations of the editing phases were taken by measuring them between the interruption point (the last segment of the reparandum) and the onset of the continuation (the first segment of the repair/continuation). In prolongations, vowel boundaries were marked between the onset and offset of the second formants of the vowels. Consonants were identified depending on their acoustic structures considering their voicing part (if any), burst, release, second formant information, and the neighborhood context, as appropriate. Prolongations were identified by the author based on her native language competence. To test statistical significance, the GLMM method, and the KruskalWallis test were used (as appropriate) using SPSS software 21.0. The confidence level was set at the conventional $95 \%$.

\section{Results}

Prolongations turned out to be the most frequent type (29\%), followed by PwWs (28.5\%), Ph-errors $(17.3 \%)$, re-starts $(12.8 \%)$, and finally false starts (12.4\%). PwWs had three subtypes according to the interruption point. Out of all PwWs 59.5\% occurred between stems and suffixes, $21.4 \%$ within the stem, while $19.1 \%$ of them were compounds with the pause 
between the two constituents (for example, gyerekek /pause/ röl 'children /pause/ about'; ver /pause/ senyez 'com /pause/ pete'; csecsemö /pause/ otthon 'infant /pause/ nursing home', respectively). Real errors occurred in one third of all disfluencies. The frequency of the five disfluencies turned out to be 0.88 incidents per minute (prolongations: 0.26 , restarts: 0.11, Ph-errors: 0.15, PwWs: 0.25, false starts: 0.10 per minute) with significant differences in the occurrences of various types of disfluencies (ChiSquare $=252.813, p=0.001$ ). Halt command was received after articulating various numbers of segments from 1 to 9 (see Figure 3).

The occurrence of all disfluencies according to pronounced number of segments shows a decrease $(27.1 \%, 18.7 \%, 18.0 \%, 17.4 \%, 13.9 \%, 10.7 \%, 5.6 \%$, $4.0 \%, 2.7 \%$, respectively). The distribution of the incidents across the quantities of abandoned words are characteristic of the disfluency types (Figure 2). The larger the pronounced part of the word the less frequent the occurrence of the disfluency.

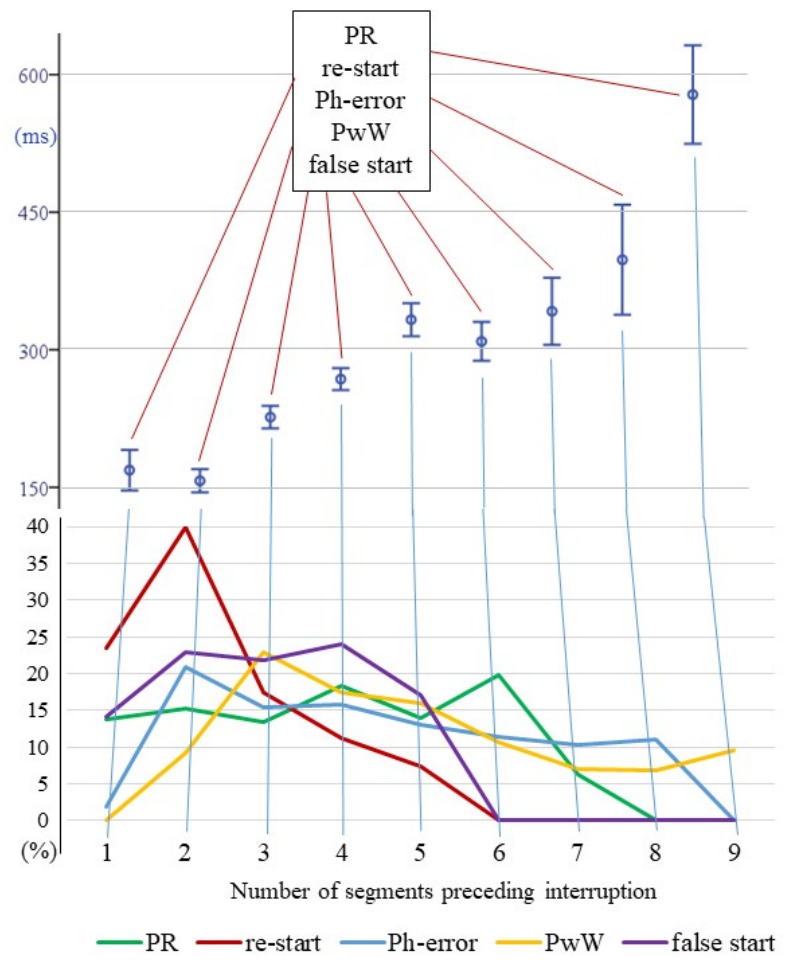

Figure 2. Occurrences of disfluency types depending on the pronounced segments prior to interruption.

No interruptions in words were found in $13.5 \%$ of all incidents of re-starts, false starts and PwWs (there were no significant differences among them). Durations of editing phases were the shortest in restarts (mean $/ \mathrm{SD}=142 / 103 \mathrm{~ms})$ and Ph-errors $(161 / 107 \mathrm{~ms})$, longer in prolongations $(274 / 79 \mathrm{~ms})$ and false starts $(236 / 153 \mathrm{~ms})$, and the longest in PwWs (376/168 ms). The editing phase durations of the PwW subtypes show large differences (between stem and suffix: $424 / 179 \mathrm{~ms}$; within the stem it is $278 / 115 \mathrm{~ms}$; and before the second word of a compound it is 333/134 ms). Significant differences were confirmed in the editing phase durations among various types of disfluencies $(F(4,1471)=169.551$, $p=0.001$ ) while pairwise tests revealed that there were no significant differences between re-starts and $\mathrm{Ph}$-errors or between $\mathrm{PwW}$ subtypes where a pause occurred in stems and in compounds.

Durations show an increase as the number of pronounced segments increases before halting with the only exception of prolongations $(F(8,1471)=90.983, \quad p=0.001)$. However, no statistically confirmed durational differences were found in some cases, between 1 and 2, 5 and 6, 5 and 7, 6 and 7, and 7 and 8 pronounced segments. In general, the more segments are pronounced prior to halt the longer the editing phases (Figure 3).

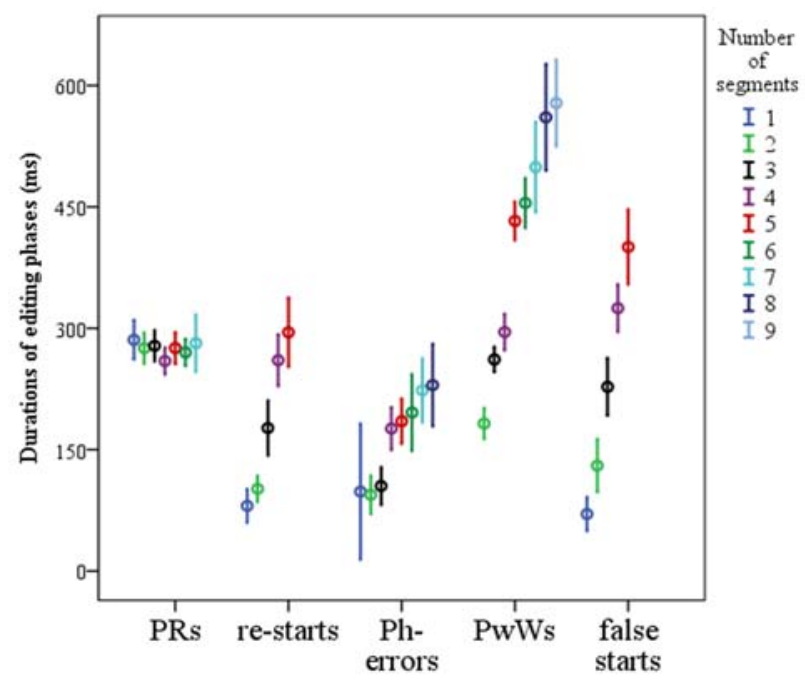

Figure 3. Durations of editing phases depending on both the disfluency type and the number of pronounced segments prior to interruption.

Up to three articulated segments before halting result in a relatively short (below $300 \mathrm{~ms}$ ) editing phase. From the fourth segment, editing phase durations show a sharp increase, particularly in PwWs. The distribution of editing phase durations seems to be characteristic of the disfluency type.

\section{Discussion}

This study focused on editing phase durations in five types of disfluencies using a large Hungarian spontaneous speech database. Findings showed that both the interruption points in the words and the durations of the editing phases and their interrelations are characteristic of the analyzed disfluencies. 
We assumed that halt command in word articulation would reflect the type of disfluency. Data supported the hypothesis. The point when articulation is interrupted indicates the speed of action decision after the monitor signals some trouble. This speed seems to be the fastest in restarts, followed by Ph-errors (Figure 2). The difference between them after the third segments before interruption is explained by the different nature of the problems. The number of articulated segments in re-starts decreases after the third segment: the monitor works quickly and accurately. The interruption points of $\mathrm{PwW}$ phenomena in stems and in compounds are similar to those of re-starts. The interruption points show some "delay" in the cases of false starts, the decrease can be seen after the fifth segment of the articulated word. It seems that it is easier to approve of a word start than to accept it to be false. Prolongations are distributed in the segments of word articulation similarly to the occurrences of incidents of the PwW subtype where a pause occurs between stem and suffix. The various intensity levels of the trouble signal and the speed of operating monitor are supposed to trigger the speaker's strategy to solve the problem.

Durations of editing phases support our observations based on interruption points. Repair of re-starts is relatively fast; all other disfluency phenomena require longer time. Repairs of a misarticulated segment can be done faster than in false starts where the speaker is uncertain even about lemma selection. They are in a way in connection with the undeniable advantage that the intended word is at hand, albeit erroneously, in Ph-errors. Pause within a word stem is significantly longer than pauses in re-starts: the speaker's strategy is different in the two cases. The temporal difference can be explained by both the diverse monitoring outcome and problem solution. Pauses in compounds are longer than those occurring in stems by $56 \mathrm{~ms}$ (the difference is insignificant, though), and can be explained by the semantically narrower bunch of lexemes in the latter case as opposed to the former type. In the retrieval of the second word of a compound $(\mathrm{PwW})$, the lemma level is reached but something prevents speech planning to step forward to lexeme level. We suggest that the relatively long editing phases before suffixes in $\mathrm{PwW}$ phenomena refer to monitoring not only the necessary suffixes but the whole grammatical structure of the context. Prolongations may reflect the speaker's trouble in lexical access or with competing (activated) lemmas but there are many other possible reasons resulting in prolongations. Speakers can be in need of extra time to select the appropriate suffixes, monitor what has been said, look for the next word or concept.
Based on measured values, a temporal hierarchy can be suggested starting with re-starts that have the shortest editing phases followed by Ph-errors, false starts and prolongations, and finally PwWs. To make the pattern a bit complicated, editing phases of PwWs in word stems are similar to those of false starts and prolongations, while those occurring before suffixes and after the first content word in a compound have the longest lingering periods. We need to emphasize that behind these findings there can be various speech planning and execution difficulties that may or may not concern lexical retrieval. We assumed that there would be a close interaction between the quantity of articulated segments of abandoned words and the durations of the editing phases. Data confirmed that faster realization of error or of any trouble in the speech planning mechanism leads to shorter editing phase.

\section{Conclusion}

We can conclude that (i) both the number of pronounced segments of a word and the corresponding editing phases are characteristic of a specific disfluency type, and (ii) speakers select a strategy to overcome their speech planning difficulties most economically considering the actual word retrieval, grammatical formulation of thought, and some other factors that seem to be decisive in that specific context.

\section{References}

Blackmer, E. \& J. Mitton. 1991. Theories of monitoring and the timing of repairs in spontaneous speech. Cognition 39(3): 173-194. https://doi.org/10.1016/0010-0277(91)90052-6

Boersma, P. \& D. Weenink. 2014. Doing phonetics by computer (version 5.4). http://www.praat.org/ (accessed 22 October 2014).

Gósy, M. 2012. BEA - A multifunctional Hungarian spoken language database. Phonetician 105/106: 5061.

Huettig, F. \& R. J. Hartsuiker. 2010. Listening to ourself is like listening to others: External, but not internal, verbal self-monitoring is based on speech perception. Language and Cognitive Processes 25(3): 347-374. https://doi.org/10.1080/01690960903046926

Levelt, W. J. M. 1989. Speaking. From intention to articulation. Cambridge, MA: MIT Press.

Nooteboom, S. G. \& H. Quené. 2008. Self-monitoring and feedback: A new attempt to find the main cause of lexical bias in phonological speech errors. Journal of Memory and Language 58(3): 837-861. https://doi.org/10.1016/j.jml.2007.05.003

Postma, A. 2000. Detection of errors during speech production: a review of speech monitoring models. https://doi.org/10.1016/S0010-0277(00)00090-1 